\title{
Fisiopatologia, avaliação e tratamento da disfunção erétil: artigo de revisão
}

\author{
Pathophysiology, evaluation and treatment of erectile dysfunction: review article
}

\author{
Andrey Biff Sarris ${ }^{1}$, Maki Caroline Nakamura ${ }^{1}$ Luiz Gustavo Rachid Fernandes ${ }^{1}$, \\ Rodrigo Luiz Staichak ${ }^{1}$, Alisson Ferreira Pupulim¹, Bernardo Passos Sobreiro²
}

Sarris AB, Nakamura MK, Fernandes LGR, Staichak RL, Pupulim AF, Sobreiro BP. Fisiopatologia, avaliação e tratamento da disfunção erétil: artigo de revisão/ Pathophysiology, evaluation and treatment of erectile dysfunction: review article. Rev Med (São Paulo). 2016 jan.-mar.;95(1):18-29.

RESUMO: A disfunção erétil (DE) é a incapacidade recorrente de obter e manter uma ereção que permita atividade sexual satisfatória, sendo a disfunção sexual que mais afeta os homens no envelhecimento. Estima-se que $50 \%$ dos homens acima de 40 anos apresentem essa disfunção. Como a prevalência é expressiva, torna-se necessária a realização de uma revisão da literatura sobre epidemiologia, fisiopatologia e tratamento acerca da DE, com o intuito de melhor compreensão da doença. A ereção é dependente de vários fatores como o relaxamento do músculo liso do corpo cavernoso, o aumento do fluxo arterial e a restrição do fluxo venoso de saída. Esses mecanismos podem estar prejudicados em várias doenças, sendo que, em alguns casos, a $\mathrm{DE}$ pode ser considerada como marcador precoce de problemas mais graves. Essa disfunção sexual apresenta várias etiologias: vascular, endócrina, neurológica, psicológica/psiquiátrica e relacionada às drogas ou às intervenções cirúrgicas. $\mathrm{O}$ próprio tratamento de algumas doenças de base pode ser a causa ou o agravamento da DE, como alguns anti-hipertensivos e antidepressivos. A principal explicação para a DE é a redução da disponibilidade de óxido nítrico, um importante vasodilatador. Contudo, fazem também parte de sua fisiopatologia a obstrução mecânica dos vasos sanguíneos e problemas de transmissão de estímulos neurológicos. ADE é primeiramente avaliada pelo método clínico, mas existem instrumentos avaliativos multidimensionais, sendo o Índice Internacional de Função Erétil considerado o padrão-ouro. $\mathrm{O}$ tratamento pontual para esta doença deve ser fundado na sua etiologia. Existem várias linhas de tratamento, sendo os inibidores da fosfodiesterase-5 e a psicoterapia (para DE psicogênica ou mista) os de primeira escolha, associados ou não à reposição de testosterona. Como segunda-linha, tem-se a injeção intracavernosa de substâncias vasoativas. Caso essas terapias não produzam resultado, a prótese peniana pode ser indicada.

DESCRITORES: Disfunção erétil/fisiopatologia; Diabetes mellitus; Hipertensão; Fatores de risco; Literatura de revisão como assunto.

\begin{abstract}
Erectile dysfunction (ED) is the repeated inability to get and maintain an erection to enable satisfactory sexual activity and it's the most common sexual dysfunction in aging men. It is estimated that $50 \%$ of men older than 40 years old exhibit this dysfunction. As the prevalence is significant, it is necessary to carry out a literature review of epidemiology, pathophysiology and treatment on the ED in order to better understand the disease. The erection is dependent on various factors such as smooth muscle relaxation of corpus cavernosum, increasing blood flow and restriction of venous outflow. These mechanisms may be impaired in several diseases, and, in some cases, ED can be considered as an early marker of more serious problems. This sexual dysfunction has several etiologies: vascular, endocrinological, neurological, psychological/psychiatric, drugrelated or related to surgical interventions. Even the treatment of some underlying diseases may be the cause or the worsening of ED, as some antihypertensives and antidepressants. The main explanation for erectile dysfunction is the reduction of the availability of nitric oxide, an important vasodilator. However, mechanical obstruction of blood vessels and neurological stimuli transmission problems are also part of its pathophysiology.
\end{abstract}

1. Graduandos em Medicina da Universidade Estadual de Ponta Grossa (UEPG).Emails: maki_naka_7@hotmail.com; gustavorachid9@ gmail.com; rodrigo_staichak@hotmail.com; alisson_pupulim@hotmail.com.

2. Médico Urologista e Professor Adjunto de Urologia do Departamento de Medicina da Universidade Estadual de Ponta Grossa (UEPG). Email: bsobreiro@hotmail.com.

Endereço para correspondência: Andrey Biff Sarris. Departamento de Medicina da Universidade Estadual de Ponta Grossa (UEPG). Av. General Carlos Cavalcanti, nº 4.748 - Uvaranas, Ponta Grossa, PR. CEP: 84030-900. Email: andreybiff@hotmail.com. 
ED is first evaluated by the clinical method, but there are multidimensional evaluation instruments as the International Index of Erectile Function, considered the gold standard. The treatment for this disease should be established in its etiology. There are several lines of treatment, but phosphodiesterase-5 inhibitors and psychotherapy (for psychogenic or mixed ED) are the first choice, with or without testosterone replacement.

\section{INTRODUÇ̃̃O}

$\Lambda$ disfunção erétil (DE) é a "incapacidade recorrente de obter e manter uma ereção que permita atividade sexual satisfatória" ${ }^{1-4}$, segundo definição proposta pelo National Institutes of Health Consensus Development Panel, no ano de $1993^{1,2}$. A DE não constitui uma doença, mas sim, uma manifestação sintomatológica de patologias isoladas ou associadas ${ }^{5}$.

Uma ereção normal depende do relaxamento do músculo liso do corpo cavernoso, do aumento do fluxo arterial peniano e da restrição do fluxo venoso de saída $^{[6-8]}$. Uma série de fatores interferem negativamente nesse mecanismo e estão associados à $\mathrm{DE}$, tais como hipertensão arterial sistêmica, diabetes mellitus, tabagismo, dislipidemia, doenças neurológicas, distúrbios hormonais, uso crônico de alguns medicamentos e distúrbios psicológicos ${ }^{9-13}$.

\section{EPIDEMIOLOGIA DA DISFUNÇÃO ERÉTIL}

A DE é a mais comum disfunção sexual que acomete homens após os $40 \operatorname{anos}^{1,9}$ - estima-se que mais de 100 milhões de homens no mundo tenham algum grau de $\mathrm{DE}^{1,9}$. $\mathrm{O}$ estudo de maior relevância epidemiológica sobre o assunto, devido a sua rigorosa metodologia, foi o Massachusetts Male Aging Study (MMAS), entre 1987 e 1989․ Utilizou-se uma amostra aleatória de 1290 homens, de 40 a 70 anos, em 11 cidades randomizadas do estado de Massachusetts, EUA. Os resultados apontaram uma prevalência global de DE de $52 \%$, sendo que $17 \%$ foram classificados como de grau leve, $25 \%$ de grau moderado e $10 \%$ de grau severo ${ }^{1}$. O trabalho também apontou um aumento da incidência de DE conforme o aumento da idade: 12,4 casos/1000 homens aos 40 anos, 29,8 casos/1000 homens aos 50 anos de idade e 46,4/1000 homens aos 60 anos $^{1}$. Embora seja evidente o aumento dos casos com a idade, a DE não é uma consequência inevitável do envelhecimento ${ }^{2}$.

Três grandes estudos epidemiológicos sobre a DE foram feitos no Brasil ${ }^{1}$. O primeiro deles, realizado em 2000, foi o Estudo do Comportamento Sexual do Brasileiro (ECOS) que contou com uma amostra de 2835 indivíduos, maiores de 18 anos e residentes em 7 cidades brasileiras. Dentre 1332 homens, 46,2\% queixaram-se de $\mathrm{DE}^{14}$. O segundo, o Estudo da Vida Sexual do Brasileiro (EVSB), conduzido entre 2002 e 2003, analisou 2862 homens
As a second-line treatment there is intracavernous injection of vasoactive substances. If these therapies do not produce satisfactory results, the penile prosthesis may be indicated.

KEYWORDS: Erectile dysfunction; Diabetes mellitus; Hypertension; Risk factors; Review literature as topic.

com mais de 40 anos de idade, dentre os quais a taxa de DE foi de $45,1 \%{ }^{15}$. O último estudo, realizado em 2006, denominado de Estudo Populacional do Envelhecimento (EPE), entrevistou 5751 homens com mais de 40 anos provenientes de 18 capitais e do Distrito Federal. Como resultado, 43,6\% da amostra apresentava $\mathrm{DE}^{16}$.

De acordo com os estudos pode-se admitir, portanto, que o valor em torno de $50 \%$ represente a prevalência da DE em homens acima dos 40 anos $^{1}$. Isso significa, em termos da população brasileira, aproximadamente 16 milhões de homens ${ }^{17}$.

\section{FISIOPATOLOGIA DA DISFUNÇÃO ERÉTIL}

As causas de DE podem ser classificadas como de etiologia psicológica, orgânica ou ainda uma combinação de ambas. As causas psicogênicas mais comuns incluem ansiedade de desempenho, transtornos psiquiátricos (ansiedade e depressão) e conflitos no relacionamento. Entre os fatores orgânicos, encontram-se causas vasculares, endócrinas, neurológicas, relacionadas a drogas e a intervenções urológicas ${ }^{9-11}$.

\section{Etiologia vascular}

A doença traumática arterial, a aterosclerose e a hipertensão arterial sistêmica (HAS) estão entre as principais causas de DE vasculogênica ${ }^{9-11,18}$. Segundo dados de um estudo publicado em 2001, com base no Índice Internacional de Função Erétil (IIEF-5), em pacientes hipertensos com idades variando de 34 a 75 anos, foi identificada prevalência de $68,3 \%$ de $\mathrm{DE}^{19}$. A HAS pode estar diretamente relacionada à diminuição da quantidade de óxido nítrico (NO), substância essencial para a ereção peniana ${ }^{20,21}$. A associação entre DE e HAS é muito complexa e pode envolver outros aspectos, como a interferência hemodinâmica provocada pela utilização de medicamentos anti-hipertensivos. Diante disso, é comum que o tratamento farmacológico da hipertensão cause DE no paciente ou em caso de DE já diagnosticada, piore o quadro ${ }^{21,22}$. A associação de HAS e diabetes mellitus dobra as chances de desenvolvimento de $\mathrm{DE}^{23,24}$. Algumas outras doenças, como a Doença de Peyronie ou uma fratura peniana com lesão da túnica albugínea, podem impedir a oclusão venosa de saída - fator essencial para a ereção levando também à $\mathrm{DE}^{7,12}$. 


\section{Etiologia endócrina}

Entre as causas endócrinas, estão o diabetes mellitus (DM), a síndrome metabólica e as alterações dos hormônios sexuais ${ }^{10,11,18}$. Entre $35 \%$ e $75 \%$ dos diabéticos apresentam algum grau de $\mathrm{DE}^{10}$. A fisiopatologia da $\mathrm{DE}$ em diabéticos pode ser resultante de suas complicações, como doenças vasculares, hipertensão arterial sistêmica, obesidade e neuropatia ${ }^{16,25,26}$. Uma metanálise envolvendo 9123 pacientes com diabetes tipo 1 ou 2 mostrou que o baixo controle glicêmico contribuiu para as altas taxas de $\mathrm{DE}$ em diabéticos. Isso ocorre porque o alto nível glicêmico está relacionado com o surgimento de doenças vasculares periféricas, coronarianas e cerebrais. Como as artérias penianas são de pequeno calibre, a obstrução de seu lúmen pode ocorrer antes da angina pectoris tornando, assim, a DE um marcador precoce para problemas mais graves ${ }^{27}$.

No DM, a exposição prolongada à glicose leva à glicação não-enzimática de proteínas plasmáticas e das membranas celulares, que podem se depositar na camada subendotelial. Dessa forma, isso pode, de maneira direta, inativar o NO endotelial impedindo a vasodilatação endotélio-dependente ${ }^{28}$. Além disso, ocorre uma grande liberação de radicais livres que reagem com o NO gerando um potente oxidante (peroxinitrito) ${ }^{28}$.

Homens portadores de Síndrome Metabólica (SM) são mais suscetíveis a desenvolverem $\mathrm{DE}^{10,11,18,29,30}$. Uma pessoa que possui mais de três aspectos metabólicos alterados citados a seguir pode ser diagnosticada como portadora de SM: obesidade central (circunferência da cintura superior a $88 \mathrm{~cm}$ na mulher e $102 \mathrm{~cm}$ no homem), hipertensão arterial sistêmica (pressão arterial sistólica > 130 e/ou pressão arterial diastólica $>85 \mathrm{mmHg}$ ), glicemia alterada (glicemia $>110 \mathrm{mg} / \mathrm{dl}$ ) ou diagnóstico de diabetes, triglicerídeos $>150 \mathrm{mg} / \mathrm{dl}$, HDL colesterol $<40 \mathrm{mg} / \mathrm{dl}$ em homens e $<50 \mathrm{mg} / \mathrm{dl}$ em mulheres ${ }^{31-33}$. Dados de 100 homens com SM apresentaram elevada prevalência de DE, quando comparados a homens que não possuíam a síndrome $(26.7 \% \text { vs } 13 \%, p=0,03)^{34}$. No entanto, o principal aspecto da síndrome presente em homens com DE é a HAS ${ }^{29,30}$.

A obesidade visceral está associada a aumentos nos níveis de peptídeo $\mathrm{C}$, glicose e insulina, desempenhando um efeito negativo sobre os níveis de testosterona. Em obesos, há aumento da atividade da enzima aromatase, que transforma a testosterona em estradiol, no tecido adiposo. $\mathrm{O}$ aumento de estradiol na corrente sanguínea suprime a produção de testosterona por feedback negativo, levando à diminuição da lipólise e aumento da deposição de gordura abdomina ${ }^{29}$. Esse efeito é visto, por exemplo, na terapia de deprivação androgênica, realizada em pacientes com câncer de próstata.

A disfunção dos hormônios sexuais masculinos, por si só, piora a situação de pacientes com DE, principalmente quando se leva em conta a associação com a síndrome hipogonádica do homem adulto e a doença arterial cavernosa ${ }^{35}$. A testosterona e seu metabólito (5 $\alpha$-di-hidrotestosterona) estimulam a expressão do gene NOS (óxido nítrico sintetase), o que aumenta os níveis de $\mathrm{NO}$ - importante vasodilatador - no tecido erétil do corpo cavernoso $^{36-40}$. Além disso, a testosterona atua também sobre a enzima fosfodiesterase-5 (PDE-5) ${ }^{36,37}$. Quando a testosterona estimula o gene NOS, que aumenta a síntese de NO, aumenta consequentemente a ação da PDE-5 e quando a regulação da testosterona cessa para o gene NOS, cessa também para a PDE-5 $5^{36,37}$.

\section{Etiologia neurológica}

Estima-se que as causas neurológicas correspondam entre 10 a $19 \%$ dos casos de $\mathrm{DE}^{10,11,18,41}$. Entre essas causas, destacam-se: doença de Parkinson, demências, doenças desmielinizantes e lesões medulares em níveis que afetam a ereção e/ou a ejaculação ${ }^{41}$.

A doença de Parkinson (DP), que afeta $1 \%$ da população acima dos 50 anos, pode ter relação direta com a DE ou com o uso de medicação para o controle da doença (ou ambos) $)^{42}$. De acordo com Singer et al. ${ }^{43} \mathrm{em}$ um estudo sobre a prevalência de DE nesses pacientes, foi identificado que $60,4 \%$ dos pacientes com DP apresentavam DE quando comparados a $37,5 \%$ do grupo controle. A DE nestes pacientes é atribuída a vários aspectos como sintomas motores, depressão, ansiedade, disfunções autonômicas e efeitos principais ou colaterais dos medicamentos antiparkinsonianos ${ }^{43}$. O déficit de dopamina na DP pode ser um possível mecanismo de associação da doença e da $\mathrm{DE}$, visto que a regulação central da ereção é dependente da estimulação dopaminérgica. Isto poderia ser confirmado a partir da observação de que o uso de apomorfina, um agonista dopaminérgico, pode induzir a ereção nesses pacientes ${ }^{44,45}$. Os níveis de testosterona também têm importante papel na regulação da ereção e esse hormônio se encontra diminuído nos pacientes com Parkinson ${ }^{46}$.

A lesão medular também se associa à $\mathrm{DE}$ - a ereção é dependente de uma série de eventos tanto a nível do sistema nervoso central quanto do periférico ${ }^{41}$. O estímulo sensitivo para a ereção é conduzido pelo nervo pudendo até o centro reflexo da medula, que contém o sistema nervoso autônomo parassimpático, cujo estímulo leva à vasodilatação das artérias penianas, permitindo um maior fluxo de sangue para os corpos cavernosos. Já o estímulo psicogênico é gerado no sistema nervoso central através de estímulos visuais, táteis e fantasias sexuais. Para um melhor desempenho da ereção, esses dois estímulos agem em conjunto, porém quando há lesão medular acima do cone medular sem afetar a cauda equina, não há ereção psicogênica, persistindo a reflexa. Já com uma lesão no cone medular ou na cauda equina, não haverá a ereção reflexa, podendo persistir a psicogênica ${ }^{41,47}$.

\section{Etiologia relacionada a drogas}

Algumas drogas também estão envolvidas no desencadeamento da $\mathrm{DE}^{10,11}$. Muitos estudos relacionam 
tabagismo à DE. Alguns dados epidemiológicos indicam que fumantes podem ter uma prevalência de DE duas vezes maior quando comparados a não-fumantes devido a alterações morfológicas do endotélio e ao estresse oxidativo produzido sobre as células ${ }^{19}$. O uso prolongado da nicotina também induz uma diminuição crônica da síntese e da disponibilidade de óxido nítrico (NO). Hart e Meston ${ }^{19}$ elaboraram um estudo longitudinal com indivíduos do sexo masculino, submetidos a uma terapia de reposição nicotínica através de adesivos transdérmicos. Ao final de oito semanas, resultados significativos foram observados na melhoria do fluxo sanguíneo, rigidez e tumescência peniana, confirmando a hipótese de que a nicotina dificultava respostas genitais masculinas independente da gravidade da DE que os pacientes apresentavam no início do estudo ${ }^{48}$. Conforme Gades et al. ${ }^{49}$, a magnitude da DE quando relacionada ao tabagismo não tem uma relação direta com a idade, podendo ocorrer em indivíduos mais jovens ${ }^{49}$.

Em relação ao álcool, doses pequenas inicialmente promovem um aumento da ereção e do desejo sexual devido ao seu efeito vasodilatador. No entanto, grandes quantidades dessa substância podem causar sedação, diminuir a libido e causar uma disfunção sexual transitória ${ }^{7}$. O alcoolismo crônico pode resultar em disfunção hepática, um decréscimo dos níveis de testosterona e um aumento dos níveis de estrogênio, podendo apresentar também casos de polineuropatia alcoólica que afeta diretamente a inervação peniana? .

\section{Etiologia relacionada à intervenção urológica}

A DE é muito comum em intervenções e afecções urológicas, sendo o câncer de próstata um importante fator de predisposição a esse evento ${ }^{50}$. A principal causa de DE em pacientes com câncer de próstata é a prostatectomia, que pode reduzir a função sexual em até $60 \%$ dos pacientes submetidos a cirurgia dentro de 2 anos e chegar a níveis de $80 \%$ em 8 anos $^{51}$. A disfunção sexual nesse caso, além de relação com o procedimento cirúrgico, pode se relacionar com outros fatores, tais como o psicológico, quando o paciente recebe diagnóstico de câncer e a idade dos pacientes, que geralmente é maior que $50 \operatorname{anos}^{52}$. Por outro lado, a radioterapia, um método mais utilizado para pacientes jovens e para tumores bem localizados, pode ser uma alternativa à prostatectomia radical ${ }^{52}$. A curto prazo, pacientes que realizaram a prostatectomia radical têm duas vezes mais chance de desenvolver DE que aqueles que fizeram o tratamento por radioterapia, porém a longo prazo (15 anos), não haveria diferença significativa entre os dois grupos ${ }^{51}$.

A hiperplasia prostática benigna (HPB) é uma das afecções mais comuns de próstata, tendo sua prevalência potencialmente relacionada com o aumento da idade ${ }^{53,54}$. A exteriorização clínica da HPB se dá pela síndrome do trato urinário baixo (LUTS - Low Urinary Tract
Symptoms), que também pode ser característica de outras patologias, de origem infecciosa, por exemplo. A LUTS pode ser classificada em irritativa e obstrutiva. A primeira se manifesta com baixo jato urinário, gotejamento e retenção urinária e a segunda com perda da complacência vesical e hipocontratilidade da musculatura detrusora, cursando com polaciúria e nictúria ${ }^{53-55}$. Além de compartilharem o mesmo grupo etário, HPB-LUTS e DE têm relações fisiopatológicas, com diminuição significativa da qualidade de vida. As consequências da síndrome metabólica, como o processo aterosclerótico em vasos penianos, levam a uma produção alterada de prostanoides que culminam com a diminuição ainda maior da síntese de óxido nítrico, o que favorece a DE e o crescimento prostático ${ }^{56,57}$. Rosen et al. ${ }^{58}$ demonstraram em um estudo com mais de 14 mil homens entre 50 e 80 anos demonstrou, através da aplicação do Índice Internacional de Função Erétil (IIEF), que o LUTS é fator de risco independente para $\mathrm{DE}^{58}$. A ressecção transuretral de próstata é considerada o padrão-ouro entre as terapias cirúrgicas para a HPB, levando à melhora dos sintomas em até $85 \%$ dos casos, mas complicações como a DE podem ocorrer em até $12 \%$ dos pacientes submetidos ${ }^{59}$.

\section{MÉTODOS DE AVALIAÇÃo PARA A DISFUNÇÃO ERÉTIL}

O primeiro passo para a avaliação da DE é o método clínico $^{10,12,13}$. Após apresentação da queixa, o médico deve investigar o histórico sexual do paciente e de sua(seu) parceira(o) (preferências sexuais, ansiedade quanto ao desempenho sexual, repertório sexual, atração pela(o) parceira(o), conflito no relacionamento, entre outros $)^{10,12,13}$. A isso, deve-se seguir o rastreamento de comorbidades e fatores de risco para DE na tentativa de associar a possível etiologia e recomendar o melhor tratamento ${ }^{10,12,13}$. Adicionalmente, podem-se utilizar métodos avaliativos padronizados $^{10,12,13}$.

Entre os instrumentos avaliativos multidimensionais propostos para avaliação da DE, os mais utilizados são o Quociente Sexual Masculino (QSM) e o Índice Internacional de Função Erétil (IIEF). Este último é considerado padrãoouro para a avaliação ${ }^{60}$. O IIEF é composto por 15 questões, agrupadas em cinco domínios: função erétil, orgasmo, desejo sexual, satisfação sexual e satisfação geral ${ }^{60-62}$. O questionário, entretanto, foi desenvolvido apenas para avaliação da função sexual de homens heterossexuais ${ }^{60}$. Os intervalos da pontuação permitem classificar a gravidade da DE em cinco grupos: Sem DE (26-30), DE leve (22$25)$, DE leve a moderada (17-21), DE moderada (11-16) e DE grave (6-10). Um estudo com o objetivo de análise do IIEF, abordando 108 pacientes, demonstrou uma taxa interessante de sensibilidade e de facilidade para aplicação do teste ${ }^{60}$. Há uma versão resumida do IIEF, chamada de IIEF-5, composta por 5 questões ${ }^{61}$. O IIEF-5 mostrou-se uma ferramenta mais objetiva e tanto sensível e eficaz 
quanto a versão integral do $\mathrm{IIEF}^{63}$.

Já o QSM é um questionário breve desenvolvido para avaliar a função e a satisfação sexual masculina ${ }^{64}$. É composto por 10 questões que abrangem os seguintes domínios da função sexual: desejo, autoconfiança sexual e autoestima, qualidades da ereção e do controle da ejaculação, capacidade de atingir o orgasmo, satisfação geral do indivíduo e de sua parceira com as preliminares e o intercurso. A partir das 10 questões, respondidas em uma escala que varia de 0 a 5 , o valor é multiplicado por 2 gerando um índice que varia de 0 a 100 . Resultados entre 82-100 indicam bom a excelente desempenho/satisfação sexual, 62-80, regular a bom, 42-60 desfavorável a regular, 22-40, ruim a desfavorável, 0-20, nulo a ruim ${ }^{64}$. A realização do QSM auxilia no direcionamento da entrevista clínica a fim de se ter uma avaliação mais correta dos problemas do paciente. A partir dele, pode-se escolher a melhor abordagem possível, trabalhando com outros especialistas. Também é um instrumento para avaliação da eficácia do tratamento das disfunções sexuais masculinas. O QSM foi construído com base em uma pesquisa realizada com 612 homens que moravam na cidade de São Paulo-BR por meio da qual foram questionados sobre os fatores que influenciavam a qualidade da vida sexual desses homens. Posteriormente, o QSM foi validado comparativamente, sendo uma ferramenta acessível aos médicos ${ }^{64}$.

\section{TRATAMENTO DA DISFUNÇÃO ERÉTIL}

O tratamento da DE deve ser, de início, de conscientização do paciente sobre a patologia, formas de tratamento e resultados esperados ${ }^{10}$. Pacientes que possuem morbidades relacionadas ao aparecimento de DE devem realizar controle destas a fim de diminuir os episódios de disfunção. $\mathrm{O}$ controle dos fatores de risco é o princípio do tratamento não-farmacológico ${ }^{7,9,10}$. A etiologia da DE também é um importante fator a se considerar para o tratamento, já que se relaciona com a escolha da linha de trabalho ${ }^{7,65}$. Geralmente, o tratamento medicamentoso tem de ser associado ao não-farmacológico logo de início9 . A primeira linha para tratamento de DE é composta pela mudança dos hábitos de vida, aconselhamento, psicoterapia/terapia sexual e os fármacos inibidores da fosfodiesterase- $5^{3,66,67}$.

1) Mudanças de hábitos de vida e aconselhamento

As mudanças de hábitos de vida e aconselhamento constituem um conjunto de medidas como o controle do sedentarismo, estresse, obesidade, uso de drogas ilícitas, tabagismo, alcoolismo, entre outros, com finalidade de melhorar a função erétil ${ }^{68,69}$. Confirmando a resolutividade desse manejo, um estudo randomizado realizado com 110 homens obesos com idades entre 35 e 55 anos, estimulados a reduzir pelo menos $10 \%$ do peso corporal com dieta diária de 1700 calorias e exercícios semanais de até 195min/ semana, demonstrou melhora no escore do IIEF de 13,9 para 17 (classificação: de "DE moderada" para "DE leve a moderada"), com 31\% dos homens recuperando a função sexual $^{27}$.

\section{2) Psicoterapia/terapia sexual}

A psicoterapia no tratamento da DE configura-se como uma abordagem ampla ao casal, dirigindo-se não somente ao portador de DE. Essa prática consiste em diversas intervenções psicológicas com o intuito de eliminar pensamentos ou mitos que afetam a relação conjugal, tendo como objetivo explorar aspectos como experiências sexuais prévias, ansiedade, entre outras. Aborda também temas pontuais e pessoais que possam interferir, como: distúrbios psiquiátricos, atividades profissionais, contexto familiar, educação sexual, entre outros. A psicoterapia tem se mostrado eficiente para o alívio dos sintomas quando o diagnóstico médico exclui causas orgânicas ${ }^{70}$.

\section{3) Inibidores da fosfodiesterase-5 (pde-5)}

Os primeiros estudos relacionando inibidores da fosfodiesterase-5 (PDE-5) e DE foram realizados pela empresa Pfizer ainda em 1993 e publicados em 1996. Em 1998, a Food and Drug Administration (FDA) aprovou o uso de citrato de Sildenafil - a primeira droga oral aprovada para $\mathrm{DE}^{65,71-73}$. Ao longo dos anos, novos inibidores foram desenvolvidos - Tadalafil $\left(\right.$ Cialis $^{\circledR}$ ) e Vardenafil $\left(\text { Levitra }^{\circledR}\right)^{71}$, ambos aprovados pelo FDA em 2003 ${ }^{65}$. Atualmente, Lodenafil $\left(\right.$ Helleva $\left.^{\circledR}\right)$ e Udenafil $\left(\right.$ Zydena $\left.^{\circledR}\right)$ também estão disponíveis no Brasil.

Diversos estudos clínicos demonstraram uma taxa de sucesso no uso de Sildenafil entre $70 \%$ a $90 \% \%^{3,72}$. A ação do Sildenafil foi demonstrada através de pletismografia peniana, com uma duração da rigidez $60 \%$ maior em relação ao placebo $^{73}$. Foi realizado um estudo prospectivo, comparativo, tipo caso-controle, no qual cada paciente era seu próprio controle, com 29 pacientes, com idades entre 32 e 75 anos e com queixa de $\mathrm{DE}^{72,73}$. Os pacientes utilizaram três comprimidos de Sildenafil 50mg antes de relações sexuais em casa por 15 dias e um comprimido $1 \mathrm{~h}$ antes do acompanhamento com os pesquisadores. Os resultados não demonstraram diferença estatística significativa de aumento do fluxo nas artérias cavernosas, provavelmente pelo número pequeno da amostra, no entanto, $72,4 \%$ dos pacientes demonstraram resposta positiva quanto à ereção. Se avaliados apenas os pacientes abaixo de 50 anos, a resposta foi de $86,67 \%{ }^{73}$.

\section{- Mecanismo de ação}

A estimulação sexual provoca uma estimulação do sistema nervoso parassimpático, que leva à liberação de óxido nítrico (NO), o qual leva ao aumento da produção de Monofosfato de Guanina Cíclica (GMPc) $)^{9,72,74,75}$. Este causa o relaxamento do músculo liso do corpo cavernoso através da redução das concentrações intracelulares de cálcio e, por 
Sarris AB, et al. Fisiopatologia, avaliação e tratamento da disfunção erétil.

fim, o aumento do fluxo arterial da região, produzindo a ereção ${ }^{75}$. Em condições normais, o GMPc é degradado pela enzima fosfodiesterase do tipo 5 (PDE-5). Os inibidores da fosfoidesterase-5 agem, como o próprio nome diz, como inibidores seletivos da PDE-5 $5^{71,72,74-76}$. Já que o GMPc não é degradado devido à inibição da PDE-5, ocorre um aumento de sua concentração, permitindo a desfosforilação das cadeias leves miosínicas e consequentemente, relaxamento do músculo liso, levando à ereção ${ }^{64}$. Como a inibição é seletiva e temporária, os inibidores não afetam a ereção em momentos nos quais não se realiza o uso da medicação ${ }^{64}$.

Apesar de os miócitos ventriculares serem dependentes de PDE-1 (e não de PDE-5), a vasodilatação arterial e venosa e a possível hipotensão arterial são preocupações com o uso dos inibidores ${ }^{72,76}$. O Sildenafil, um dos inibidores, tem uma pequena seletividade, em média, 1000 vezes menor para as PDE-2 (encontradas principalmente no córtex adrenal), PDE-3 (músculo liso, plaquetas e miocárdio) e PDE-4 (linfócitos do cérebro e do pulmão). Apresenta também, uma seletividade moderada (80 vezes menor) para as PDE-1 (localizadas no músculo liso, rins e cérebro) e uma seletividade ainda maior para as PDE-6, encontradas nas células fotorreceptoras da retina ${ }^{75}$.

- Comparação entre os três primeiros Inibidores da PDE-5: Sildenafil, Tadalafil e Vardenafil

No Brasil, o Sildenafil (Viagra ${ }^{\circledR}$ ) existe nas dosagens 25, 50 e 100mg; o Vardenafil (Levitra ${ }^{\circledR}$ ), nas dosagens 5, 10 e $20 \mathrm{mg}$ e o Tadalafil $\left(\right.$ Cialis $^{\circledR}$ ) nas doses de 5 e $20 \mathrm{mg}$ e o Lodenafil $\left(\right.$ Helleva $^{\circledR}$ ), na dose $80 \mathrm{mg}$. As principais diferenças entre os três inibidores (Sildenafil, Vardenafil e Tadalafil) são a potência de inibição da enzima e as propriedades farmacocinéticas, como velocidade de absorção, meia-vida plasmática e duração do efeito (Tabela 1$)^{3}$.

Tabela 1. Comparação entre Sildenafil, Vardenafil e Tadalafil ${ }^{3,7,9,74-76}$

\begin{tabular}{l|c|c|c}
\hline & Sildenafil & Vardenafil & Tadalafil \\
\hline Tempo para Cmáx - horas & 0,8 & $0,7-0,9$ & 2 \\
\hline Início da ação - minutos & $15-60$ & $15-60$ & $15-120$ \\
\hline Meia-Vida - horas & $3-5$ & $4-5$ & Sem efeito \\
\hline Comidas gordurosas & Reduzem a absorção & Reduzem a absorção & \multicolumn{2}{c}{ Sim } \\
\hline Efeitos adversos: & \multicolumn{1}{|c}{ Sim } & Sim \\
\hline \multicolumn{1}{c|}{ - Cefaleia, dispepsia e flush cutâneo } & Sim & Raro & Raro \\
\hline \multicolumn{1}{c|}{ - Mialgia e dor lombar } & Raro & Raro & Não \\
\hline Precaução visual com uso de antiarrítmicos & Sim & Sim & Sim \\
\hline Contraindicação com uso de nitratos & Não & Sim & \\
\hline
\end{tabular}

O "Estudo Observacional da Disfunção Erétil" (EDOS) foi um estudo prospectivo observacional, longitudinal e com 6 meses de duração, entre 2003 e 2004, realizado em 9 países europeus ${ }^{66}$. O objetivo do estudo foi avaliar a satisfação com o tratamento para DE, a mudança de tratamento e outros fatores associados. Os pacientes foram divididos em três grupos - uso de Sildenafil, uso de Tadalafil e uso de Vardenafil - e foram avaliados 3 e 6 meses após a inclusão. Nesse estudo, não houve diferença estatística significativa entre a melhora da ereção entre os três grupos tanto no acompanhamento de 3 meses quanto no de 6 meses $^{66}$.

Os efeitos colaterais costumam ser transitórios e de leve intensidade. Os mais frequentes são: cefaleia, rubor facial, dispepsia e congestão nasal. Dor lombar e mialgia são mais frequentes com o uso do Tadalafil ${ }^{65,72,74}$. A cefaleia e o rubor são provavelmente produzidos pela vasodilatação dos leitos vasculares cerebrais e cutâneos, respectivamente. Outro efeito adverso possível dos inibidores de PDE-5 é uma perda de visão transitória ou até mesmo permanente, devido a uma afecção denominada neuropatia óptica isquêmica não-arterítica. Não há certeza sobre o mecanismo e o papel dos inibidores nessa afecção ${ }^{76}$. Nota-se em estudos que o Tadalafil tem ação menor sobre os inibidores da PDE-6 (retinianos) em relação ao Sildenafil e Vardenafil, mostrando assim, uma redução da probabilidade de efeitos colaterais da visão em relação aos outros dois $(0,1 \%$ para o Tadalafil contra $3 \%$ do Sildenafil $)^{71}$. Entretanto, o efeito da perda de visão é transitório e raro ${ }^{71}$.

Os inibidores da PDE-5 são metabolizados primordialmente pela via do citocromo CYP3A4. Sendo assim, inibidores deste citocromo (Ritonavir, Saquinavir etc.) e outras drogas metabolizadas por essa via (Cetoconazol) retardam a metabolização dos inibidores, aumentando seus níveis plasmáticos ${ }^{3}$.

- Inibidores da PDE-5 e Doenças Cardiovasculares

Devido à alta prevalência de $\mathrm{DE}$, os efeitos adversos dos inibidores da PDE-5, em especial sobre o sistema cardiovascular, sempre foram uma grande preocupação. 
Estudos indicaram não-significância para aumento do risco de eventos cardiovasculares graves com o uso ocasional de inibidores da PDE-5 mesmo em pacientes com angina estável e outras doenças cardiovasculares. Para portadores de hipertensão arterial sistêmica e que usam antihipertensivos, diversas recomendações existem em relação aos cuidados do uso de inibidores da PDE-5, devido ao risco de hipotensão arterial ${ }^{74,76}$. Uma das contraindicações absolutas dos inibidores da PDE-5 é o uso concomitante de nitratos, pois os inibidores da PDE-5 prolongam seus efeitos vasodilatadores, causando extrema hipotensão reflexa ${ }^{64,65,72,74,76}$. Estabelece-se uma diferença mínima de $24 \mathrm{~h}$ para uso dos medicamentos ${ }^{76}$.

Queixas quanto à relação uso de inibidores da PDE5 e eventos cardiovasculares graves - tais quais infarto agudo do miocárdio e acidente vascular encefálico - foram relatadas ao FDA no ano de $1999^{74}$. A averiguação do FDA estipulou que dados estavam faltando nos prontuários, como a história médica do paciente, dosagem dos inibidores da PDE-5 e os dados conclusos de causa de morte - o que não permitiu uma associação direta ${ }^{74}$.

\section{- Inibidores da PDE-5 e Perda Auditiva}

Mais de 90 casos de perda auditiva associados a inibidores da PDE-5 já foram reportados, a partir de 2007. Um estudo experimental sul-coreano de 2008 em ratos mostrou os potenciais efeitos de perda auditiva com uso prolongado e de altas doses de inibidores de PDE-577-79. O mesmo ocorreu em um estudo clínico de $2009^{[80]}$. Um estudo de coorte de 2010, avaliando 11.525 homens, com 40 anos ou mais, por 2 anos, mostrou que a perda auditiva foi duas vezes mais comum entre utilizadores dos inibidores de PDE-5 ${ }^{82}$. Mais estudos a respeito são necessários, mas alguma relação já é observada e precisa ter a atenção médica $^{78,80,81}$.

Quarenta e sete casos de perda de audição súbita relacionada aos inibidores da PDE-5 foram investigados por agências de farmacovigilância. Os dados dos indivíduos com perda auditiva mostraram uma idade média de 56,6 anos, sendo que $88 \%$ destes tiveram perda unilateral e em mais de $50 \%$ dos casos o Sildenafil era o fármaco envolvido. Salienta-se também que nos casos apresentados a surdez mesmo pós-terapia foi atenuada, mas irreversível ${ }^{78}$.

\section{- Novos Inibidores da PDE-5}

Existem outras drogas inibidoras seletivas da PDE-5 apresentadas nos últimos anos ${ }^{65}$. O carbonato de Lodenafil $\left(\right.$ Helleva $^{\circledR}$ ) foi desenvolvido no Brasil pela Cristália Produtos Químicos e Farmacêuticos e já está sendo comercializado. Seu tempo de duração é de cerca de 6 horas, com tempo de meia vida de 1,6h $( \pm 0,4)$. Um de seus diferenciais é a capacidade de não alterar a pressão arterial, frequência cardíaca ou alterações no intervalo QT com o uso simultâneo de álcool ${ }^{83}$. O Udenafil $\left(\right.$ Zydena $\left.^{\circledR}\right)$ é um potente inibidor desenvolvido pela sul-coreana Dong
Companhia Farmacêutica e também já está em uso no Brasil, no entanto, ainda não foi aprovado pela FDA ou pela Agência de Medicamentos Europeia (EMEA) ${ }^{84}$. Com uma concentração máxima relativamente rápida $(1,0-1,5 \mathrm{~h})$ e um tempo de meia vida de 11 a $13 \mathrm{~h}$, o Udenafil parece uma boa opção para apenas uma tomada/dia ${ }^{85}$.

Outro sul-coreano é o Mirodenafil (Mvix $\left.{ }^{\circledR}\right)$, também já em comercialização na Coreia do Sul. Um dos mais promissores inibidores da PDE-5 é o Avanafil, desenvolvido pela japonesa Mitsubishi Tanabe Companhia Farmacêutica e que demonstrou eficácia favorável, segurança e farmacocinética ${ }^{86}$. O Avanafil foi aprovado pelo FDA em abril de 2013 e pela EMEA em junho 2013. Atualmente a norte-americana Vivus Companhia Farmacêutica é quem está desenvolvendo o Avanafil ${ }^{65}$.

\section{4) Injeção intracavernosa}

Um dos problemas dos inibidores da PDE-5 é a pouca ou nenhuma resolutividade que esses medicamentos têm para homens com impotência por lesão medular ou outra lesão de inervação e para homens que carecem de libido. Para os pacientes não responsivos aos tratamentos orais ou que apresentem contraindicação a eles, valese do tratamento de segunda linha da DE, a injeção intracavernosa, que gera poucos efeitos adversos mesmo após 8 anos de uso pelo paciente ${ }^{75,87,88}$. O mecanismo de combate à DE é a injeção de substâncias vasoativas como a prostaglandina E1 (PGE1), papaverina, fentolamina e clorpromazina no corpo cavernoso do pênis, que resulta em vasodilatação peniana levando à ereção. Atualmente, só o alprostadil (análogo da PGE1) é liberado para esse fim. Como efeitos colaterais, podem-se citar dor ou desconforto local pós-aplicação, hematomas, priaprismo e, tardiamente, fibrose local, sendo a injeção intracavernosa contraindicada para pacientes que tenham história de coagulopatias severas, priaprismo de repetição ou doenças cardiovasculares mal controladas ${ }^{87}$.

\section{5) Terapia hormonal}

O hormônio testosterona pode ser utilizado como terapia medicamentosa para tratamento da DE, principalmente associado aos inibidores da PDE-5. A associação de testosterona propicia além do efeito vascular da ereção peniana, um aumento da libido e da tumescência noturna em pacientes que tenham comprometimento das células de Leydig ${ }^{35,39}$.

Entretanto, a terapia de reposição de testosterona em homens não é o tratamento de primeira linha para a $\mathrm{DE}$, principalmente porque a $\mathrm{DE}$ depende muito pouco da redução dos efeitos deste hormônio ${ }^{89,90}$. A testosterona melhora a libido proporcionando maior desejo para se ter uma relação sexual, entretanto, nem todos os pacientes que tenham índices de testosterona abaixo do normal sofrem influência em seu desejo sexual através da manipulação da testosterona ${ }^{35,38,90}$. No estudo de Aversa et al ${ }^{89}$, os pacientes 
possuíam níveis reduzidos de androgênios, mas não abaixo do limiar inferior. Assim, a administração de testosterona a esses pacientes não alterou a libido, pois o limiar desse hormônio necessário à libido é extremamente baixo. Esse estudo concluiu que a melhora da função sexual se deveu aos efeitos vasculares causados pela administração de testosterona ${ }^{89}$.

Spittzer et al. ${ }^{91}$ realizaram um estudo comparativo entre dois grupos de pacientes que utilizavam Sildenafil para DE associado ou não à reposição de testosterona. Os autores não encontraram diferença entre os grupos e sugeriram que o Sildenafil pode atuar no mecanismo de aumento da testosterona por si só ${ }^{91}$. Sendo assim, a utilização da reposição de testosterona para tratamento de DE depende de vários fatores e inclui a discussão sobre os riscos e benefícios com o paciente. O guia de prática clínica de Bhasin et a. ${ }^{92}$, recomenda que a administração de testosterona só se dê em pacientes que possuam níveis de testosterona total e livre abaixo do limiar normal, 315 $\mathrm{ng} / \mathrm{dL}$ para testosterona total e $6,25 \mathrm{ng} / \mathrm{dL}$ para testosterona livre, associados a sintomas de deficiência androgênica, como por exemplo, DE. A redução fisiológica dos níveis de testosterona ocorre principalmente em pacientes idosos, na velocidade 1-2\% por ano. Essa redução ocorre principalmente após os 80 anos e caracteriza o distúrbio androgênico do envelhecimento masculino (DAEM) ${ }^{93}$.

Os sintomas clássicos da DAEM encontrados na maioria dos pacientes são: diminuição da função sexual, podendo ocorrer casos de $\mathrm{DE}$, redução do crescimento de cabelo e barba, diminuição da massa muscular e massa óssea e redução da libido ${ }^{92,94}$. Entretanto, a utilização deste hormônio deve ser repensada devido aos diversos efeitos adversos, como policitemia, hiperplasia prostática benigna, sintomas severos do trato urinário inferior e apneia obstrutiva do sono. Como o câncer de próstata é hormôniodependente, a administração de testosterona estimula o crescimento do tumor e predispõe ao aparecimento de metástases $^{92}$.

A principal forma de administração é através de ésteres de testosterona injetáveis. Estão disponíveis no Brasil: propionato, fenilpropionato, cipionato, decanoato, isocaproato e undecanoato. A medicação deve ser repetida a cada 2 ou 3 semanas para medicamentos de curta duração e a cada 6 ou 12 semanas para aqueles de longa duração $0^{95}$.

A utilização de reposição hormonal oral se dá principalmente pelo undecanoato de testosterona, com doses de $160 \mathrm{mg} /$ dia, embora não haja relatos de que sua administração melhore a função sexual do paciente ${ }^{95}$. $\mathrm{O}$ undecanoato de testosterona também foi administrado via intramuscular tendo melhora parcial em alguns estudos e nenhuma melhora em outros ${ }^{96-99}$. A administração de dehidroepiandrosterona (DHEA) $50 \mathrm{mg}, 2$ vezes ao dia, pode ser feita, contudo não há estudos que documentem sua eficácia. Ainda como medicamento oral, mucoadesivos bucais com $30 \mathrm{mg}$ tem tido eficácia em melhorar os níveis de testosterona em pacientes hipogonádicos ${ }^{100-102}$. Outra forma de administração de testosterona é a utilização de adesivos transdérmicos. Os adesivos escrotais liberam de 4 a $6 \mathrm{mg} /$ dia de testosterona e são melhor absorvidos que os não-escrotais. Em pacientes com DAEM, os adesivos transdérmicos são bem aceitos na dose de 5 a 7,5 mg/dia e trocados a cada 48 horas $^{100-102}$. Os adesivos não-escrotais liberam $5 \mathrm{mg} /$ dia de testosterona e devem ser aplicados em pele glabra, seca e limpa. Os géis também são absorvidos via transdérmica. São disponibilizados na concentração de $1 \%$ e a dose inicial é de $5 \mathrm{~g} / \mathrm{dia}$, podendo ser expandida para $10 \mathrm{~g} /$ dia. Devem ser aplicados em pele não exposta, dorso, abdome ou braços ${ }^{95,100-102}$.

\section{6) Prótese peniana}

Em caso de insucesso das terapias de primeira e segunda linhas, pode-se optar por uma terapia de terceira linha, como a prótese peniana ${ }^{2}$. Na maior parte dos casos, esse tipo de tratamento é tido como a última opção ${ }^{102}$. As próteses penianas são "esqueletos" artificiais que ajudam a manter a ereção do pênis. A prótese semirrígida consiste em um tubo flexível de silicone com uma haste interna de prata ou platina que confere tensão ao pênis ${ }^{103}$. Essa estrutura é introduzida no corpo cavernoso e pode assumir três posições: linha reta para os atos sexuais; para baixo para urinar; posição normal para ficar oculta sob as roupas. As próteses infláveis possuem um mecanismo hidráulico para o funcionamento. Estas são compostas por cilindros flexíveis que se localizam nos corpos cavernosos ligados a uma bomba com função reservatório que fica no escroto, acima da túnica dartos ${ }^{103}$. Para obter uma ereção, o indivíduo aperta algumas vezes o escroto para que o soro saia do reservatório e encha os cilindros. Para obter o efeito contrário, deve-se dobrar os cilindros para baixo ${ }^{103}$. Há um tipo de prótese inflável mais moderna que contêm três elementos: cilindros, bomba e reservatório abdominal ${ }^{103}$.

As próteses semirrígidas são mais baratas e de fácil manipulação pelo paciente. A vantagem da inflável é a qualidade estética, porém exige maior habilidade manual para manipular o escroto. Uma das complicações de ambas as próteses é o risco de infecção, cujos índices oscilam entre $1,3 \%$ e 4,3\%. Acrescido a esse risco existem as complicações cirúrgicas que podem chegar a até $8 \%$. Na prótese inflável também há o risco de falhas mecânicas que variam entre 9 e $13 \%{ }^{103}$, apesar de terem um período de sobrevida de 5 anos $^{2,103}$.

\section{CONCLUSÃO}

A disfunção erétil (DE) apresenta alta prevalência estando relacionada a faixas etárias mais avançadas e apresentando relação com outros eventos associados à saúde. É, portanto, expressão da condição da saúde masculina. Esses fatos se tornam preocupantes quando enquadrados na situação brasileira de transição demográfica e epidemiológica. 
A grande prevalência de doenças que culminam na DE torna este quadro frequente tanto na população mundial quanto na brasileira. Isso torna importante a detecção e a avaliação da DE, visto o impacto que essa condição desempenha na qualidade de vida do indivíduo. Apesar de existirem vários mecanismos ainda obscuros de como

\section{REFERÊNCIAS}

1. Riedner CE. Avaliação do efeito da obesidade na associação entre doença cardíaca isquêmica e disfunção erétil [Tese]. Porto Alegre: Programa de Pós-Graduação em Medicina Ciências Médicas, Universidade Federal do Rio Grande do Sul (UFRS); 2010. Disponível em: https://www.lume.ufrgs.br/ bitstream/handle/10183/25115/000751947.pdf?sequence=1.

2. Schiavini JL, Damião R. Abordagem da disfunção erétil. Rev Hosp Univ Pedro Ernesto. 2010;9(supl.):48-59. Disponível em: http://revista.hupe.uerj.br/detalhe_artigo.asp?id=253.

3. Sociedade Brasileira de Urologia. Abdo CHN, Bertero EB, Faria GE, Torres LO, Glina S, participantes. Disfunção erétil: tratamento com drogas inibidoras da fosfodiesterase tipo 5. Projeto diretrizes: Associação Médica Brasileira e Conselho Federal de Medicina. Sociedade Brasileira de Urologia, jun. 2006. Disponível em: http://projetodiretrizes. org.br/5_volume/16-Disfun5.pdf.

4. Burnett AL. Evaluation and management of erectile dysfunction. In: Wein AJ, Kavoussi LR, Campbell MF, et al. Campbell-Walsh urology. 10th ed. Philadelphia: Elsevier; 2012. v.1, p.721-9.

5. Moura LR, Ceresér KMM. Aspectos farmacológicos do citrato de sildenafila no tratamento da disfunção erétil. Rev Bras Med. 2002;59(4):265-75. Disponível em: http://www. moreirajr.com.br/revistas.asp? fase=r003\&id_materia $=1877$.

6. Garaffa G, Minhas S, Ralph D. Male sexual function. In: Mundy AR, Fitzpatrick JM, Neal DE, et al. The scientific basis of urology. 3rd ed. London: Informa Healthcare; 2010. p.300.

7. Lue TF. Physiology of penile erection and pathophysiology of erectile dysfunction. In: Wein AJ, Kavoussi LR, Campbell MF, et al. Campbell-Walsh urology. 10th ed. Philadelphia: Elsevier; 2012. v.1, p.688-720.

8. Viaro F, Nobre F, Evora PRB. Expressão de óxido nítrico sintetases na fisiopatologia das doenças cardiovasculares. Arq Bras Cardiol. 2000;74(4):365-79. Disponível em: http:// publicacoes.cardiol.br/abc/2000/7404/74040009.pdf.

9. Wyllie M. As drogas e o sistema genitourinário. In: Page C, Curtis M, Sutter M, et al. Farmacologia integrada. 2a ed. Barueri: Manole; 2004. p.495-501.

10. McVary KT. Sexual dysfunction. In: Longo DL, Fauci AS, Kasper DL, et al. Harrison's: principles of internal medicine. 18th ed. New York: McGraw-Hill; 2012. v.1, p.374-8.

11. Swerdloff RS, Wang C. The testis and male sexual function. In: Goldman L, Schafer AI. Goldman's Cecil medicine. 24th ed. Philadelphia: Elsevier; 2012. v.1, p.1519-29.

12. Mullins JK. Erectile dysfunction and Peyronie's disease. In: Parsons K, Eifler JB, Han M. Handbook of urology. Chichester: Wiley Blackwell; 2014. p.55-64.

13. Lue TF. Male Sexual Dysfunction. In: Lue TF, McAninch JW. Smith \& Tanagho's General Urology. $18^{\mathrm{a}}$ ed. San Francisco: Mc Graw Hill Lange; 2013. p.596-627.

14. Abdo CHN. Perfil sexual da população brasileira: resultado determinadas patologias geram o quadro de $\mathrm{DE}$, há várias linhas de tratamento para essa condição, farmacológicas e não-farmacológicas. Logo, é importante conhecer a epidemiologia e a fisiopatologia dessa disfunção sexual para uma identificação e um manejo de qualidade nos pacientes com DE.

do estudo do comportamento sexual (ECOS) do brasileiro. Rev Bras Med. 2002;59(4):250-7. Disponível em: http://www. moreirajr.com.br/revistas.asp? fase=r003\&id_materia $=1875$.

15. Abdo CHN, Scanavino MT, Martins FG, Oliveira Jr WMO. Disfunção erétil: resultados do estudo da vida sexual do brasileiro. Rev Assoc Med Bras. 2006;52(6):424-9. doi: http:// dx.doi.org/10.1590/S0104-42302006000600023.

16. Abdo CHN, Afif-Abdo J. Estudo populacional do envelhecimento (EPE): primeiros resultados masculinos. Rev Bras Med. 2007;64(8):379-83. Disponível em: http://www. moreirajr.com.br/revistas.asp? fase=r003\&id_materia $=3629$.

17. Instituto Brasileiro de Geografia e Estatística (IBGE). Censo 2010: pirâmide etária - distribuição da população por sexo, segundo os grupos de idade [citado 10 mar. 2014]. Disponível em: http://www.censo2010.ibge.gov.br/sinopse/webservice/ default.php? $\operatorname{cod} 1=0 \& \operatorname{cod} 2=\& \operatorname{cod} 3=0 \&$ frm $=$ pirâmide .

18. Buzin L. Avaliação de função erétil, parâmetros androgênicos, questionários de rastreamento de hipogonadismo de início tardio e obesidade central em homens com 40 anos ou mais, participantes de um rastreamento de câncer de próstata [dissertação]. Porto Alegre: Programa de Pós-Graduação em Medicina: Ciências Médicas, Universidade Federal do Rio Grande do Sul (UFRGS); 2010. Disponível em: http://hdl. handle.net/10183/18771.

19. Teixeira AS, Pereira BJ. Disfunção erétil como marcador precoce de doença cardiovascular. Rev Factores Risco. 2012;(25):8-11. Disponível em: http://www.spc.pt/DL/RFR/ artigos/405.pdf.

20. Perticone F, Sciacqua A, Maio R, Perticone M, Maas R, Boger $\mathrm{RH}$, et al. Asymmetric dimethylarginine, L-arginine, and endothelial dysfunction in essential hypertension. J Am Coll Cardiol. 2005;46(3):518-23. doi: http://dx.doi.org/10.1016/j. jacc.2005.04.040.

21.Romero JC, Reckelhoff JF. Role of angiotensin and oxidative stress in essential hypertension. Hypertension. 1999;34(2):9439. doi: http://dx.doi.org/10.1161/01.HYP.34.4.943.

22.Grimm RH Jr, Grandits GA, Prineas RJ, McDonald RH, Lewis CE, Flack JM, et al. Long-term effects on sexual function of five antihypertensive drugs and nutritional hygienic treatment in hypertensive men and women. Hypertension. 1997;29(1):814. doi: http://dx.doi.org/10.1161/01.HYP.29.1.8.

23.Parazzini F, Menchini FF, Bortolotti A, Calabrò A, Chatenoud L, Colli E, et al. Frequency and determinants of erectile dysfunction in Italy. Eur Urol. 2000;37(1):43-9. doi: http:// dx.doi.org/10.1159/000020098.

24.Tostes RC, Carneiro FS, Lee A, Giachini FR, Leite R, Osawa $\mathrm{Y}$, et al. Cigarette smoking and erectile dysfunction: focus on NO bioavailability and ROS generation. J Sex Med. 2008;5(6):1284-95. doi: http://dx.doi.org/10.1111/j.17436109.2008.00804.x.

25.Owiredu WKBA, Amidu N, Alidu H, Sarpong C, GyasiSarpong CK. Determinants of sexual dysfunction among 
Sarris AB, et al. Fisiopatologia, avaliação e tratamento da disfunção erétil.

clinically diagnosed diabetic patients. Reprod Bio Endocrinol. 2011;70(9):7827-39. doi: http://dx.doi.org/10.1186/14777827-9-70.

26.Chitaley K, Kupelian V, Subak L, Wessells H. Diabetes, obesity and erectile dysfunction: field overview and research priorities. J Urol. 2009;182(6):45-50. doi: http://dx.doi. org/10.1016/j.juro.2009.07.089.

27.Souza CA, Cardoso FL, Silveira RA, Wittkopf PG. Importância do Exercício Físico no Tratamento da Disfunção Erétil. Rev Bras Cardiol. 2011;24(3):180-5. Disponível em: http://sociedades.cardiol.br/socerj/revista/2011_03/a_2011_ v24_n03_06impor t.pdf.

28. Wajchenberg BL. Disfunção endotelial no diabetes do tipo 2. Arq Bras Endocrinol Metab. 2002;46(5):514-9. doi: http:// dx.doi.org/10.1590/S0004-27302002000500004.

29.Traish AM, Guay A, Feeley R, Saad F. The dark side of testosterone review deficiency: I. Metabolic syndrome and erectile dysfunction. J Androl. 2009;30(1):10-22. Doi: http:// dx.doi.org/10.2164/jandrol.108.005215.

30.Vlachopoulos C, Rokkas K, Stefanadis C, Ioakeimidis N. Inflammation, metabolic syndrome, erectile dysfunction and coronary artery disease: common links. J Eur Urol. 2007;52:1590-600. doi: http://dx.doi.org/10.1016/j. eururo.2007.08.004.

31.Tomada I, Tomada N, Neves D. Disfunção eréctil: doença (cardio)vascular. Acta Urol. 2010;27(1):27-34. Disponível em: http://www.apurologia.pt/acta/1-2010/Disf-Erec-Doen-Card. pdf.

32.Zambon JP, Mendonça RR, Wroclawski ML, Karam Jr A, Santos RD, Carvalho JAM. Cardiovascular and metabolic syndrome risk among men with and without erectile dysfunction: case-control study. Sao Paulo Med J. 2010;128(3):137-40. doi: http://dx.doi.org/10.1590/S151631802010000300006 .

33. Traish AM, Haider A, Doros G, Saad F. Long-term testosterone therapy in hypogonadal men ameliorates elements of the metabolic syndrome: an observational, long-term registry study. Int J Clin Pract. 2013;68(3):314-29. doi: http://dx.doi. org /10.1111/ijcp.12319.

34.Esposito K, Giugliano F, Martedi E, Feola G, Marfella R, D'Armiento M, et al. High Proportions of Erectile Dysfunction in Men With the Metabolic Syndrome. Diabetes Care. 2005;28(5):1201-3. doi: http://dx.doi.org/10.2337/ diacare.28.5.1201.

35.Chatterjee R, Kottaridis PD, McGarrigle HH, Linch DC. Management of erectile dysfunction by combination therapy with testosterone and sildenafil in recipients of high-dose therapy for haematological malignancies. Bone Marrow Transplant. 2002;29(7):607-10. doi: http://dx.doi.org/10.1038/ sj.bmt.1703421.

36. Isidori AM, Buvat J, Corona G, Goldstein I, Jannini EA, Lenzi A, et al. A critical analysis of the role of testosterone in erectile function: from pathophysiology to treatment - a systematic review. Eur Urol. 2013;65:99-112. doi: http:// dx.doi.org/10.1016/j.eururo.2013.08.048.

37. Traish AM, Goldstein I, Kim NN. Testosterone and erectile function: from basic research to a new clinical paradigm for managing men with androgen insufficiency and erectile dysfunction. Eur Urol. 2007;52(1):54-70. doi: http://dx.doi. org/10.1016/j.eururo.2007.02.034.

38. Aversa A, Isidori AM, De Martino MU, Caprio M, Fabbrini E, Rocchietti-March M, et al. Androgens and penile erection: evidence for a direct relationship between free testosterone and cavernous vasodilation in men with erectile dysfunction. Clin Endocrinol (Oxf). 2000;53(4):517-22. doi: http://dx.doi. org/10.1046/j.1365-2265.2000.01118.x.

39. Shabsigh R. Testosterone therapy in erectile dysfunction. Aging Male. 2004;7(4):312-8. doi: http://dx.doi. org/10.1080/13685530400016540.

40. Tejada S, Ângulo J, Cellek S, González-Cadavid N, Heaton J, Pickard R, et al. Physiology of erectile function. J Sex Med. 2004;1(3):254-65. doi: http://dx.doi.org/10.1111/j.17436109.04038.x.

41. Antuña VC, Gómez FJM, Escaf S, Fernández-González F. Etiología neurógena en pacientes con disfunción erectil. Arch Esp Urol. 2008;61(3):403-11. Disponível em: http:// scielo.isciii.es/scielo.php?script=sci arttext\&pid=S000406142008000300007\&lng=es.

42.Lucon M, Pinto ASS, Simm RF, Haddad MS, Arap S, Lucon $\mathrm{AM}$, et al. Avaliação da disfunção erétil em pacientes com doença de Parkinson. Arq Neuropsiquiatr. 2001;59(3):559-62. doi: http://dx.doi.org/10.1590/S0004-282X2001000400014.

43. Singer C, Weiner WJ, Sanchez-Ramos JR. Autonomic dysfunction in men with Parkinson's disease. Eur Neurol. 1992;32(3):134-40. doi: http://doi.org/10.1159/000116810.

44.Papatsoris AG, Deliveliotis C, Singer C, Papapetropoulos S. Erectile dysfunction in Parkinson's disease. Urology. 2006;67(3):447-51. doi: http://doi.org/10.1016/j. urology.2005.10.017.

45. Argiolas A, Melis MR. Central control of penile erection: role of the paraventricular nucleus of the hypothalamus. Prog Neurobiol. 2005;76(1):1-21. doi: http://dx.doi.org/10.1016/j. pneurobio.2005.06.002.

46. Okun MS, McDonald WM, DeLong MR. Refractory nonmotor symptoms in male patients with Parkinson disease due to testosterone deficiency: a common unrecognized comorbidity. Arch Neurol. 2002;59(5):807-11. doi: http:// dx.doi.org/10.1001/archneur.59.5.807.

47. Falavigna A, Finger G, Souza OE. Spinal cord injury and male infertility: a review. Coluna/Columna. 2012;11(4):322-5. doi: http://dx.doi.org/10.1590/S1808-18512012000400015.

48. Harte CB, Meston CM. Are the inhibitory effects of nicotine on erectile response in nonsmokers generalizable to long-term smokers? A reply. J Sex Med. 2004;5(8):2003-4. doi: http:// dx.doi.org/10.1111/j.1743-6109.2008.00891.x.

49. Gades NM, Nehra A, Jacobson DJ, McGree ME, Girman CJ, Rhodes $\mathrm{T}$, et al. Association between smoking and erectile dysfunction: a population-based study. Am J Epidemiol. 2005;161(4):346-51. doi: http://dx.doi.org/10.1093/aje/ kwi052.

50. Han M, Trock BJ, Partin AW. The impact of preoperative erectile dysfunction on survival after radical prostatectomy. BJU Int. 2010;106(11):1612-7. doi: http://dx.doi.org/10.1111/ j.1464-410X.2010.09472.x.

51. Resnick MJ, Koyama T, Penson DF. Long-term functional outcomes after treatment for localized prostate cancer. N Eng J Med. 2013;368(5):436-45. doi: http://dx.doi.org/10.1056/ NEJMoa1209978.

52. Hoppe BS, Nichols RC, Henderson RH, Morris CG, Williams $\mathrm{CR}$, Costa J, et al. Erectile function, incontinence, and other quality of life outcomes following proton therapy for prostate cancer in men 60 years old and younger. Cancer. 2012;118(18):4619-26. doi: http://dx.doi.org/10.1002/ cncr. 27398 . 
53. Burnett AL, Wein AJ. Benign prostatic hyperplasia in primary care: what you need to know. J Urol. 2006;175(3):19-24. doi: http://dx.doi.org/10.1016/S0022-5347(05)00310-1.

54. Madersbacher S, Alivizatos G, Nordling J, Sanz CR, Emberton M, de la Rosette JJ. EAU 2004 guidelines on assessment, therapy and follow-up of men with lower urinary tract symptoms suggestive of benign prostatic obstruction (BPH guidelines). Eur Urol. 2004;46(5):547-54. doi: http:// dx.doi.org/10.1016/j.eururo.2004.07.016.

55. Rosen RC. Assessment of sexual dysfunction in patients with benign prostatic hyperplasia. BJU Int. 2006;97(2):29-33. doi: http://dx.doi.org/10.1111/j.1464-410X.2006.06103.x.

56. Klotz T, Mathers MJ, Bloch W, Nayal W, Engelmann Ul. Nitric oxide based influence of nitrates on micturition in patients with benign prostatic hyperplasia. Int Urol Nephrol. 1999;31:335-41.

57. Azadzoi KM, Babayan RK, Kozlowski R, Siroky MB. Chronic ischemia increases prostatic smooth muscle contraction in the rabbit. J Urol. 2003;170:659-63. doi: http:// dx.doi.org/10.1097/01.ju.0000064923.29954.7e.

58. Rosen R, Altwein J, Boyle P, Kirby RS, Lukacs B, Meuleman $\mathrm{E}$, et al. Lower urinary tract symptoms and male sexual dysfunction: the multinational survey of the aging male (MSAM-7). Eur Urol. 2003;44(6):637-49. doi: http://dx.doi. org/10.1016/j.eururo.2003.08.015.

59. Cavalcanti AGLC, Errico G, Araújo JFC, Ribeiro JGA, Scaletscky R. Hiperplasia prostática benigna. Projeto diretrizes: Associação Médica Brasileira e Conselho Federal de Medicina. São Paulo: Sociedade Brasileira de Urologia e Sociedade Brasileira de Medicina de Família e Comunidade; jun. 2006 [citado 25 ago. 2015]. Disponível em: http:// projetodiretrizes.org.br/5_volume/24-Hiperpla.pdf.

60. Gonzáles AI, Sties SW, Wittkopf PG, Mara LS, Ulbrich $\mathrm{AZ}$, Cardoso FL, et al. Validação do Îndice Internacional de Função Erétil (IIFE) para uso no Brasil. Arq Bras Cardiol. 2013;101(2):176-82. doi: http://dx.doi.org/10.5935/ abc. 20130141 .

61. Cordeiro AC, Mizzaci CC, Fernandes RM, Araujo Jr AG, Cardoso PO, Dutra LV, et al. Índice internacional de função erétil simplificado e doença coronariana em pacientes hipertensos. Arq Bras Cardiol. 2012;99(4):924-30. doi: http:// dx.doi.org/10.1590/S0066-782X2012005000081.

62. Quek KF, Low WY, Razacl AH, Chua CB, Loh CS, Dublin $\mathrm{N}$. The performance properties of the International Index of Erectile Function (IIEF-15) in assessing erectile dysfunction in patients with lower urinary tract symptoms. Med J Malaysia. 2002;57(4). Disponível em: http://www.e-mjm. org/2002/v57n4/International_Index_Erectile_Function.pdf.

63. Rhoden EL, Telöken C, Sogari PR, Souto CAV. The use of the simplified International Index of Erectile Function (IIEF5 ) as a diagnostic tool to study the prevalence of erectile dysfunction. Int J Impot Res. 2002;14:245-50. doi: http:// dx.doi.org/10.1038/sj.ijir.3900859.

64. Abdo CH. The male sexual quotient: a brief, self-administered questionnaire to assess male sexual satisfaction. J Sex Med. 2007;4(2):382-9. doi: http://dx.doi.org/10.1111/j.17436109.2006.00414.x.

65. Katzung BG, Chatterjee K. Vasodilatadores e tratamento da angina de peito. In: Katzung BG. Farmacologia - básica \& clínica. 9a ed. Rio de Janeiro: Guanabara Koogan; 2005. p.159.

66. Codevilla CF, Castilhos TS, Bergold AM. A review of analytical methods for the determination of four new phosphodiesterase type 5 inhibitors in biological samples and pharmaceutical preparations. Braz J Pharm Sci. 2013;49(1):1-11. doi: http://dx.doi.org/10.1590/S198482502013000100002 .

67. Morales AM, Hernández PRG, Rico FM, Arrondo JLA, Disla T. Efectividad y satisfacción com el tratamiento en pacientes con disfunción eréctil em España: estudio EDOS. Actas Urol Esp. 2010;34(4):356-64. doi: http://dx.doi.org/10.1016/j. acuro.2010.02.018.

68. Abdo JA. Diagnóstico e tratamento da disfunção erétil. Diagn Trat. 2007;12(4):192-5. Disponível em: http://files.bvs.br/ upload/S/1413-9979/2007/v12n4/a0017.pdf.

69. Gupta BP, Murad H, Clifton MM, Prokop L, Nehra A, Kopecky SL. The effect of lifestyle modification and cardiovascular risk factor reduction on erectile dysfunction a systematic review and meta-analysis. Arch Intern Med. 2011;171(20):1797-803. doi: http://dx.doi.org/10.1001/ archinternmed.2011.440.

70. Costa JFLV. Abordagem psicoterapêutica no tratamento da disfunção eréctil [dissertação]. Porto, Portugal: Instituto de Ciências Biomédicas Abel Salazar. Universidade do Porto; 2010. Disponível em: http://repositorioaberto.up.pt/bitstream/10216/52797/2/Abordagem $\% 20$ Psicoteraputica\%20no\%20Tratamento\%20da\%20 Disfuno\%20Erctil.pdf.

71. Smith VB, Spina D, Page CP. Phosphodiesterase inhibitors. Br J Pharmacol. 2006;147:252-7. doi: http://dx.doi. org/10.1038/sj.bjp.0706495.

72. Shinlapawittayatorn K, Chattipakorn S, Chattipakorn N. Effect of sildenafil citrate on the cardiovascular system. Braz J Med Biol Res. 2005;38:1303-11. doi: http://dx.doi. org/10.1590/S0100-879X2005000900003.

73. Claro JA, Ximenes SF, Andrade E. Effect of sildenafil in cavernous arteries of patients with erectile dysfunction. International Braz J Urol. 2003;29(4):320-6. doi: http:// dx.doi.org/10.1590/S1677-55382003000400006.

74. Andersen ML, Bignotto M, Tufik S. Effect of sildenafil $\left(\right.$ Viagra $\left.^{\circledR}\right)$ on the genital reflexes of paradoxical sleepdeprived male rats. Braz J Med Biol Res. 2007;40:1473-80. doi: http://dx.doi.org/10.1590/S0100-879X2006005000180.

75. Guimarães AC, Malachias MVB, Coelho OR, Zilli EC, Luna RL. Use of Sildenafil in Patients with Cardiovascular Disease. Arq Bras Cardiol. 1999;73(6):521-6. doi: http:// dx.doi.org/10.1590/S0066-782X1999001200006.

76. Chong DY, Michel T. Farmacologia do tônus vascular. In: Golan DE, Tashjian AH, Armstrong EJ, Armstrong AW. Princípios de farmacologia: a base fisiopatológica da farmacoterapia. 2a ed. Rio de Janeiro: Guanabara Koogan; 2009. p.346-7.

77. Mukherjee B, Shivakumar T. A case of sensorineural deafness following ingestion of sildenafil. J Laryngol Otol. 2007;121(4):395-7. doi: http://dx.doi.org/10.1017/ S0022215106005512.

78. Khan AS, Sheikh Z, Khan S, Dwivedi R, Benjamin E. Viagra deafness - sensorineural hearing loss and phosphodiesterase-5 inhibitors. Laryngoscope. 2011;121(5):1049-54. doi: http:// dx.doi.org/10.1002/lary.21450.

79. Hong BN, Yi TH, Kim SY, Kang TH. High dosage sildenafil induces hearing impairment in mice. Biol Pharm Bull. 2008;31(10):1981-4. doi: http://dx.doi.org/10.1248/ bpb.31.1981. 
80.Maddox PT, Saunders J, Chandrasekhar SS. Sudden hearing loss from PDE-5 inhibitors: a possible cellular stress etiology. Laryngoscope. 2009;119(8):1586-9. doi: http://dx.doi. org/10.1002/lary.20511.

81.McGwin G Jr. Phosphodiesterase type 5 inhibitor use and hearing impairment. Arch Otolaryngol Head Neck Surg. 2010;136(5):488-92. doi: http://dx.doi.org/10.1001/ archoto.2010.51.

82.Barreto MASC, Bahmad F. Phosphodiesterase type 5 inhibitors and sudden sensorineural hearing loss. Braz $\mathrm{J}$ Otorhinolaryngol. 2013;79(6):727-33. doi: http://dx.doi. org/10.5935/1808-8694.20130133.

83.Silva AC, Toffoletto O, Lucio LAG, Santos PF, Afiune JB, Massud FJ, et al. Repercussão cardiovascular, com e sem álcool, do carbonato de lodenafila, um novo inibidor da PDE5. Arq Bras Cardiol. 2010;9(2):160-7. doi: http://dx.doi. org/10.1590/S0066-782X2010000200004.

84.Paick JS, Kim SW, Yang DY, Kim JJ, Lee SW, Ahn TY, et al. The efficacy and safety of udenafil, a new selective phosphodiesterase type 5 inhibitor, in patients with erectile dysfunction. J Sex Med. 2008;5(4):946-53. doi: http://dx.doi. org/10.1016/j.eururo.2008.07.025.

85.Kang SG, Kim JJ. Udenafil: efficacy and tolerability in the management of erectile dysfunction. Ther Adv Urol. 2013;5(2):101-10. doi: http://dx.doi. org/10.1177/1756287212470019.

86.Kedia GT, Ückert S, Assadi-Pour F, Kuczyk MA, Albrecht K. Avanafil for the treatment of erectile dysfunction: initial data and clinical key properties. Ther Adv Urol. 2013;5(1):35-41.

87.Simões FA, Castilho LN, Acqua LZD, Neto KAT, França WA, Iatarola DL. Como diagnosticar e tratar disfunção erétil. Rev Bras Med. 2012;69(3):39-48. Disponível em: http://www.moreirajr.com.br/revistas.asp?fase $=$ r003\&id materia $=4967$

88.Turner LA, Althof SE, Levine SB, Risen CB, Bodner DR, Kursh ED, et al. Self-injection of papaverine and phentolamine in the treatment of psychogenic impotence. J Sex Marital Ther. 1989;15(3):163-76. doi: http://dx.doi. org/10.1080/00926238908403820.

89. Aversa A, Isidori AM, Spera G, Lenzi A, Fabbri A. Androgens improve cavernous vasodilation and response to sildenafil in patients with erectile dysfunction. Clin Endocrinol (Oxf). 2003;58(5):623-8. doi: http://dx.doi.org/10.1046/j.1365$2265.2003 .01764 \mathrm{x}$

90.Shabsigh R. Hypogonadism and erectile dysfunction: the role for testosterone therapy. Int J Impot Res. 2003;15(4):9-13. doi: http://dx.doi.org/10.1038/sj.ijir.3901030.

91.Spitzer M, Bazaria S, Travison TG, Davda MN, Paley A, Cohen B, et al. Effect of testosterone replacement on response to sildenafil citrate in men with erectile dysfunction. Ann Intern Med. 2012;157(10):681-91. doi: http://dx.doi. org/10.7326/0003-4819-157-10-201211200-00004.

92.Bhasin S, Cunningham GR, Hayes FJ, Matsumoto AM, Snyder PJ, Swerdllof RS, et al. Testosterone therapy in men with androgen deficiency syndromes: an endocrine society clinical practice guideline. J Clin Endocrinol Metab. 2006;91(6):19952010. doi: http://dx.doi.org/10.1210/jc.2009-2354.

93.Nigro N, Christ-Crain M. Testosterone treatment in the aging male: myth or reality? Swiss Med Wkly. 2012;142:w.13539. doi: dx.doi.org/10.4414/smw.2012.13539.

94.Kaufman JM, Vermeulen A. The decline of androgen levels in elderly men and its clinical and therapeutic implications. Endocrine Rev. 2005;26(6):833-76. doi: http://dx.doi. org/10.1210/er.2004-0013.

95.Martits AM, Costa EMF, Nardi AC, Nardoza Jr A, Facio Jr J, Faria G, et al. Hipogonadismo masculino tardio (andropausa): tratamento. Projeto diretrizes: Associação Médica Brasileira e Conselho Federal de Medicina. Sociedade Brasileira de Endocrinologia e Metabologia. São Paulo; jul. 2004 [citado 15 ago. 2015]. Disponível em: http://www.projetodiretrizes. org.br/projeto_diretrizes/063.pdf.

96.Jockenhövel F, Minnemann T, Schubert M, Freude S, Hübler D, Schumann C, et al. Comparison of long-acting testosterone undecanoate formulation versus testosterone enanthate on sexual function and mood in hypogonadal men. Eur J Endocrinol. 2009;160(5):815-9. doi: http://dx.doi.org/ 10.1530/EJE-08-0830.

97.Yassin AA, Saad F. Improvement of sexual function in men with late-onset hypogonadism treated with testosterone only. J Sex Med. 2007;4(2):497-501. http://dx.doi.org/ 10.1111/j.1743-6109.2007.00442.x

98.Emmelot-Vonk MH, Verhaar HJ, Nakhai-Pour HR, Grobbee DE, van der Schouw YT. Effect of testosterone supplementation on sexual functioning in aging men: a 6-month randomized controlled trial. Int J Impot Res. 2009;21(2):129-38. http:// dx.doi.org/ 10.1038/ijir.2009.5.

99.Morales A, Black A, Emerson L, Barkin J, Kuzmarov I, Day A. Androgens and sexual function: a placebocontrolled, randomized, double-blind study of testosterone vs. dehydroepiandrosterone in men with sexual dysfunction and androgen deficiency. Aging Male. 2009;12(4):104-112. http://dx.doi.org/ 10.3109/13685530903294388.

100. Ebert T, Jockenhövel F, Morales A, Shabsigh R. The current status of therapy for symptomatic late-onset hypogonadism with transdermal testosterone gel. Eur Urol. 2005;47(2):13746. doi: http://dx.doi.org/10.1016/j.eururo.2004.09.015.

101. Katznelson L, Robinson MW, Coyle CL, Lee H, Farrell CE. Effects of modest testosterone supplementation and exercise for 12 weeks on body composition and quality of life in elderly men. Eur J Endocrinol. 2006;155:867-75. doi: http:// dx.doi.org/10.1530/eje.1.02291.

102. Palmas A, Coelho MF, Cardoso P, Fonseca J. Prótese peniana maleável: avaliação do grau de satisfação do doente e da parceira - experiência do Serviço de Urologia do Hospital Fernando Fonseca no período de 2005 a 2008. Acta Urol. 2009;26(1):13-8. Disponível em: http://www.apurologia.pt/ acta/1-2009/prot-pen-mal.pdf.

103. Claro JA, Arruda H, Srougi M. Alterações da função sexual masculina. Sinopse Urol. 2003;7(2):41-8. Disponível em: http://www.moreirajr.com.br/revistas.asp? fase=r003\&id materia $=2298$. 